\title{
EFFECT AND EFFECT CHAIN IN FUNCTIONAL DESIGN
}

\author{
Guozhong Cao ${ }^{1}$, Haixia Guo ${ }^{2}$, Runhua Tan ${ }^{1}$ \\ 'School of Mechanical Engineering, Hebei University of Technology, Tianjin, China. Email: \\ cgzghx@163.com; ${ }^{2}$ School of Management, Hebei University of Technology, Tianjin, China.
}

\begin{abstract}
Functional design is a key step in the product design process, whether original or redesign. To push functional design into the realm of repeatable and computational, this paper proposes an automated functional design approach. The realization of function is supported by effect chain which is produce by effects using six effect modes. This approach can produce numerous feasible principle solutions in conceptual design process. A design example for functional design of a Chinese medicine mechanism is presented to demonstrate the proposed functional design methodology.
\end{abstract}

Key words: Effect, TRIZ, Functional Design

\section{INTRODUCTION}

Functional design in engineering design research theory is a wellresearched and active field of engineering study ${ }^{1}$. Functional design plays the central role in ensuring design quality and product innovation.

Theory of Inventive Problem Solving (TRIZ) is an integration of concepts, principles, methods and cases which are abstracted by analyzing patents of the world wide. According to Altshuller's patent search, for any given problem, there is more than a $90 \%$ of chance that a similar problem has already been addressed somewhere, at some time ${ }^{2} \cdot \operatorname{Tan}^{3}$ proposed a process of two stages analogy-based design employing TRIZ, in which the process of determining TRIZ special solution from a domain problem is called the first stage analogy process which can perform automatically. The

Please use the following format when citing this chapter:

Cao, Guozhong, Guo, Haixia, Tan, Runhua, 2006, in International Federation For Information Processing (IFIP), Volume 207, Knowledge Enterprise: Intelligent Strategies In Product Design, Manufacturing, and Management, eds. K. Wang, Kovacs G., Wozny M., Fang M., (Boston: Springer), pp. 412-420. 
question now becomes: how the appropriate information can be accessed and organized?

Although there are now some general methodologies dealing with functions or reasoning about functions ${ }^{1,4-7}$, few computational tools exist to assist designers during the functional design, and functional design success is often heavily weighted on personal experience and innate ability. To push functional design into the realm of repeatable and computational, this paper proposes an automated functional design approach that utilizes six effect modes and the existing effect knowledge base to support functional realization.

\section{FUNCTIONAL DESIGN BASED ON BEHAVIOR AND EFFECT}

$\mathrm{Cao}^{8}$ proposes a functional design approach based on behavior and effect. Function is a statement to describe the transformation of input and output flows, aiming to achieve the designer's purpose. Function is expressed as verb. The 30 functions in TRIZ are expanded and reclassified and the standard set of functions is presented. The object moving between functions is called a flow, which is divided into material, energy and parameter flows based upon the work by Pahl and Beitz. Flow is expressed as noun. The standard set of flows is presented. Each flow contains a set of attributes to describe its state; two flows are different if their attributes are not the same. The standardized sets of functions and flows are intended to comprehensively describe the design space.

Behavior is a causal relationship between input and output flow. The transformation of input and output flows performs function. Function is an abstracted and subjective representation of behavior, and behavior is a physical interpretation of function. The difference in function and behavior only lies in the identification of their input and output. From this observation, it is shown that function and behavior are different views of the same thing.

The function types derived from the relation between input and output flows identify the behavior. The behaviors, which characterize the implementation of function, are called external behaviors. As with most complex system, it is generally good to break large external behaviors down into smaller and more easily sub-behaviors. An internal behavior is a sequence of alternating sub-behaviors and sub-behaviors transitions, which represents the way of external behavioral achievement. The causal behavioral reasoning based on effect in TRIZ is to support functional modeling. The mapping from function to structure is realized by relationship between effects and examples based on the analysis of patents. An example 
contains structural feature, such as what elements the design is composed of, what the attributes of the elements are and how they are related.

The functional design approach based on behavior and effect provides an automatic computational solution to complex conceptual design systems. Due to the mappings among function, behavior, effect and example are many-to-many relationship, many principle solutions are usually produced. For the multi-solutions, it is needed to determine one or a few domain solutions through evaluation. The result of detailed design is design files (drawings or data file) that can be used for manufacture.

\section{EFFECT AND EFFECT CHAIN IN FUNCTIONAL DESIGN}

\subsection{Effect}

Effect is one of the knowledge base tools in TRIZ. By the analysis of hundreds of thousands patents, effects are emerged from the relevance between behaviors delivered by a design product described in a patent and a principle used in the product ${ }^{5}$. An Effects is an input and output relationship that combines the laws of science including physics, mathematics, chemistry and geometry, and their corresponding engineering applications, which helps to bridge the gap between science and engineering. According to the included information effects can be divided into physical, chemical, geometric effects, etc.

Effects can be characterized by its input, output relations ${ }^{9}$. Generally speaking, an effect has an input and output flow, which is called as basic effect, thus the effect model has two poles, as shown in Figure 1(a). Most transitions from input to output with effect are controlled by auxiliary flow, so the controllable effect should be denoted with three poles, as shown in Figure 1(b). The control flow specifies the factors that can be manipulated to change the output intensity of an effect.

An effect may have multiple input flows, output flows or control flows, so the effect has multiple input poles, output poles or control poles (two, three or more). This paper only discusses effects with less than three input flows, output flows, or control flows.

Effects can fulfill transition from inputs to output, namely, the happening of sub-behaviors depends upon effects. When an effect is said to fulfill a given sub-behavior, it implies that there exists at least an effect, such that the sub-behavior can be achieved. 


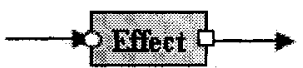

(a) Effect model with two poles

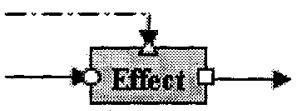

(b) Effect model with three poles

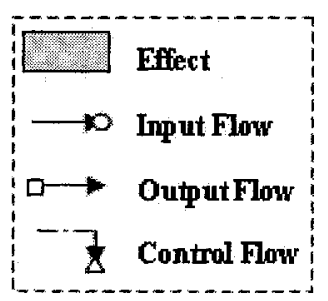

Figure 1. Effect

\subsection{Effect Mode}

Effects can be connected to one another through its input or output ports and compatible relationships among adjacent effects, which confirm causal relation and structural relation of sub-behaviors. The internal behavior can be achieved by the following effect modes, in which the directed link represents one or several flows.

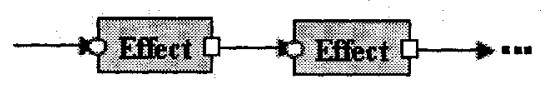

(a) Serial effect mode

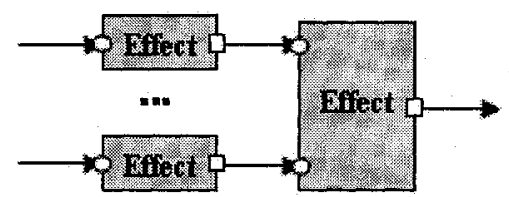

(b) Parallel effect mode

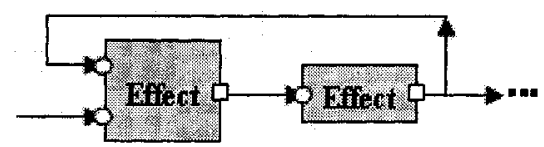

(c) Ring effect mode

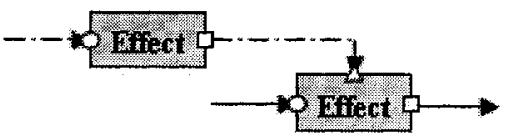

(d) Control effect mode

Figure 2. Effect modes

- Single effect mode: achieve an internal behavior by an effect, namely, the internal behavior only includes single sub-behavior, as shown in Figure 1(a). An effect can fulfill several behaviours. A behavior can be fulfilled by several effects respectively.

- Serial effect mode: achieve an internal behavior by a set of effects occurring in sequence, as shown in Figure 2(a).

- Parallel effect mode: achieve an internal behavior by a set of effects occurring at same time, as shown in Figure 2(b).

- Ring effect mode: achieve an internal behavior by a set of effects, as shown in Figure 2(c). 
- Control effect mode: the internal characteristic of an effect can be controlled by other effects in order to control the achievement mode of internal behavior, as shown in Figure 2(d).

- Combined effect mode: achieve an internal behavior by several above effects modes.

\subsection{Effect Chain}

The effects can be linked into an effect chain by using effect modes. This is useful when you do not know how to produce the input required by an effect you want to use. Multiple effect chains that produce the same output can be generated, allowing you to then select the chain that best fits your available resources and interrelated constraints. The effect chain can help engineers achieve breakthrough innovation by proposing new and unexpected variations in producing a specific output.

To generate an effect chain, only those effects where the output flow matches the input flow of the next one can be linked. The consistency between input of the first effect and output of the next one can be denoted by $\mathrm{D}_{\mathrm{c}}$ (Degree of consistency). Supposed there are $m$ attributes in required output flow, and there are $n$ attributes in produced output flow which satisfy the attributes in budget output flow $(n \leqslant m)$. $\mathrm{D}_{\mathrm{c}}$ can be shown as:

$$
D_{c}=\frac{n}{m} \times 100 \%
$$

The effects chosen for an effect chain must be compatible with each other, that is, the name of output flows of the first effect must be same as that of input flow of the next one and $D_{c}=100 \%$.

During the transformation from input flow to output flow by effect modes, there are three methods, namely, method of exhaustion, method of minimal path length and method of consistent degree.

- Method of exhaustion

If times of transformation from input flow to out flow are unlimited, there will be theoretically uncountable effect chains which produce the same output. Figure 3 shows two reasoning model for effect chain: forward direction reasoning model and backward direction reasoning model.

In forward direction reasoning model, first of all identify the input flow $\left(f_{i}\right)$ and search for the effects whose input is consistent with the input flow $\left(f_{i}\right)$. Then according to each effect the output flow $\left(f_{\text {pol }}\right)$ is produced. If the name of produced output flow is the same as required output flow (Name: $f_{p o l}=f_{q}$ ) and $D_{c}$ $=100 \%$, an effect chain is generated and the path length is evaluate as one $(p=1)$. When the first reasoning is over $\left(t_{r}=1\right)$ or all the produced output flows don't match required output flow, take the produced output flow $\left(f_{p o l}\right)$ as new input 
flow and carry through the second reasoning $\left(t_{r}=2\right)$. Repeat the above process until the reasoning times $\left(\mathrm{t}_{\mathrm{r}}=\mathrm{n}\right)$ designer permits.

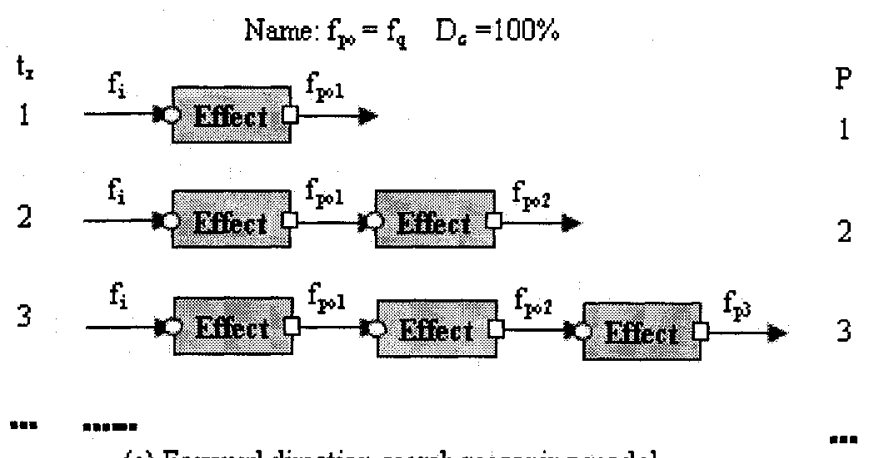

(a) Forward direction search reasoning model

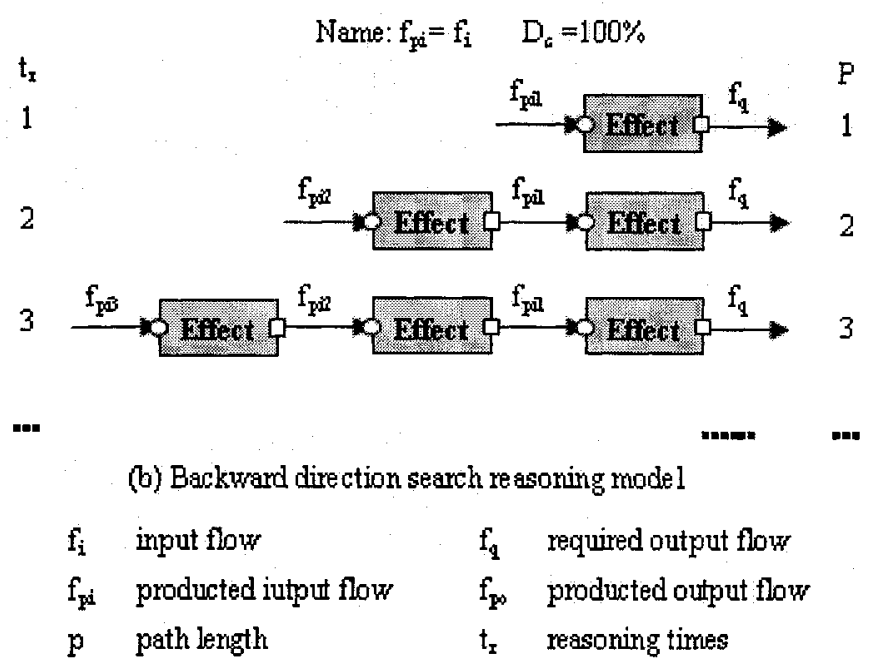

Figure 3. Reasoning model for effect chain

In backward direction reasoning model, first of all identify the required output flow $\left(f_{q}\right)$ and search for the effects whose output is consistent with the required output flow $\left(f_{q}\right)$. Then according to each effect the input flow $\left(f_{i_{1}}\right)$ is produced. If the name of produced input flow is the same as input flow (Name: $\left.f_{p i l}=f_{i}\right)$ and $D_{c}=100 \%$, an effect chain is generated and the path length is evaluate as one $(p=1)$. When the first reasoning is over $\left(t_{r}=1\right)$ or all the produced input flows don't match input flow, take the produced input flow $\left(f_{\text {pil }}\right)$ as new output flow and carry through the second reasoning $\left(t_{r}=2\right)$. Repeat the above process until the reasoning times $\left(t_{\mathrm{r}}=n\right)$ designer permits. 
Method of exhaustion can produce multiple effect chains in large-scale effects, which offer various potential principle solutions and are helpful for product innovative design, but the computational of method of exhaustion is complex and inefficient.

- Method of minimal path length

In principle, the numbers of effects that consist of effects chains can be chosen according to user discretion. But the effects chains should be as short as possible in order to obtain a simple system. The path of between two flows can be identified by method of exhaustion. The minimal path length $\left(p_{m}\right)$ is the minimum value of path length of transformation between two flows. The knowledge of minimal path length $\left(k_{p}\right)$ is presented by input flow $\left(f_{i}\right)$, output flow $\left(f_{o}\right)$ and minimal path length $\left(p_{m}\right)$, as shown in the following:

$$
k_{p}=\left\{f_{i}, f_{o}, p_{m}\right\}
$$

For example, $\{E, \Delta 1,1\}$ denotes that the minimal path length from electric field (E) to length change $(\Delta I)$ is one (by electrostriction effect).

Designers can rapidly achieve transformation from input flow to required output flow by the knowledge of minimal path length $\left(\mathrm{k}_{\mathrm{p}}\right)$. The method is high performance, but maybe the effect chain couldn't satisfy the requirement.

\section{- Method of consistent degree}

In the method of exhaustion, each reasoning process produces various effects. If degree of consistency $\left(D_{c}\right)$ between produced output flow of an effect and required output flow or between produced input flow of an effect and input flow is figured out, the produced output/input flow whose $D_{c}$ is maximum can be identified and used as new input/output flow and carry through the next reasoning. Thus the method of consistent degree is more effective than the method of exhaustion.

\section{CASE STUDY}

Pill is a kind of good form of Chinese traditional medicine, but it can not be produced by Western medicine facility for its process and physics characteristic. The present condition is long process, high energy consume and great labor intension, so it is important to develop continue forming and shorten process to meet the need of modern times.

The granulator system can be initially modeled as a black-box in Figure 4, whose inputs are powder (medicinal powder) and liquid (cementing liquid), and whose outputs are sphericity and particle (pill).

According to the known inputs and outputs, search for the effects by forward/backward direction reasoning models. In order to shorten manufacturing process of pill, the reasoning times is set as three $\left(t_{r}=4\right)$. The 
effects can be automatically linked into effect chains by using effect modes. Figure 5 shows the part of effect chains of granulator system, which are mainly based on fluidized bed effect, vibration effect, plastic deformation effect, shear effect, friction effect and Pascal's effect. The solution structure of granulator system based on Figure 5(d) is shown in Figure 6.

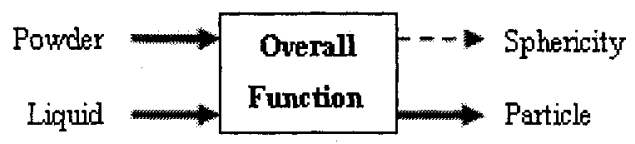

Figure 4. Black-box model of granulator system

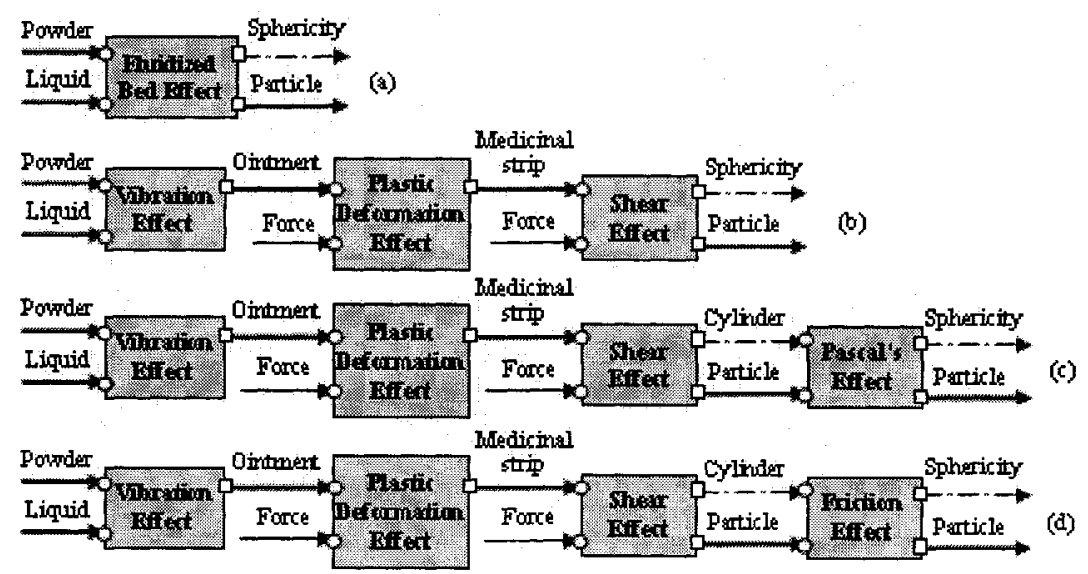

Figure 5. Effect chains of granulator system

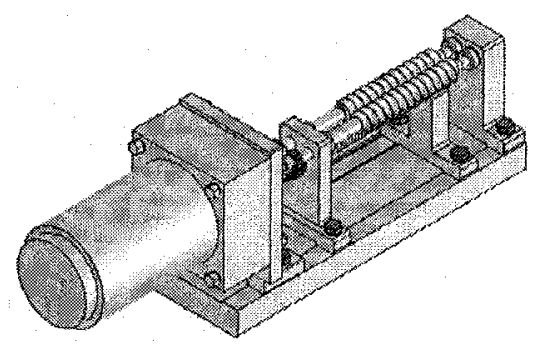

Figure 6. Principle solutions of granulator system 


\section{CONCLUSION}

Conceptual design confirms the principle solution that contains the required function. Functional design is a process in conceptual design that impacts the key features of the design result.

This paper proposes an automated functional design approach that utilizes six effect modes and the existing effect knowledge base to support functional realization. Based on effect modes, the Effect Module in computer-aided innovation design software InventionTool 2.0 has been developed. This approach can produce numerous feasible principle solutions in conceptual design process.

A design example for functional design of a Chinese medicine mechanism is presented to demonstrate the proposed functional design methodology and prove that the method is feasible.

\section{ACKNOWLEDGEMENTS}

The research is under the support of Natural Science Foundation of China (No. 50375045) and Natural Science Foundation of Tianjin (No. 043802211).

\section{REFERENCE}

1. Stone, R. B. and Wood, K. L., (1998), Development of a functional basis for design, Transactions of the ASME, Journal of Mechanical Design, vol.122, pp.359-370.

2. Altshuller, G., (1999), The Innovation Algorithm, TRIZ, Systematic Innovation and Technical Creativity, Technical Innovation Center, INC, Worcester.

3. Tan, R., (2005), Process of two stage analogy-based design employing TRIZ, The $1^{\text {st }}$ IFIP Working Conference on Computer Aided Innovation, ULM, Germany.

4. Pahl, G. and Beitz, W., (1996), Engineering Design - A Systematic approach, The 2nd Edition, Springer-Verlag London.

5. Altshuller, G., (1984), Creativity as an Exact Science, Gordon and Branch Publishers, Luxembourg.

6. Umeda, Y., Ishii, M., Yoshioka, M., et al., (1996), Supporting conceptual design based on the function-behavior-state modeler, Artificial Intelligence for Engineering Design, Analysis and Manufacturing: Aiedam, vol.10, pp. 275-288.

7. Qian, L. and Gero, J. S., (1996), Function-Behavior-Structure Paths and their Role in Analogy-based Design, AIEDAM, vol10, pp. 289-312.

8. Cao G., Tan R., Zhang R., et al., (2005), Study on Conceptual Design Process Model Based on Effects, China Mechanical Engineering, vol.16, pp.823-827. (in Chinese)

9. Tan R., (2002), Innovation Design-TRIZ: Theory of Innovative Problem Solving, China Mechanic Press. (in Chinese) 